\title{
PROOF OF BLASCHKE'S SPHERE CONJECTURE
}

\author{
BY LEON W. GREEN ${ }^{1}$
}

Communicated by Hans Samelson, October 17, 1960

Let $M$ be a $C^{2}$, compact, orientable two-dimensional Riemannian manifold with positive Gaussian curvature $R(x), x \in M$. Assume that the distance between a point $x$ of $M$ and the first point conjugate to $x$ on any geodesic ray emanating from $x$ is independent of the initial direction and of $x$. We shall prove the following conjecture of Blaschke:

\section{THEOREM. $M$ has constant curvature.}

For a brief history of the problem and a proof under stringent additional conditions see [2].

Let us first recall some of the known facts about such surfaces which we shall use. Proofs may be found in $[1, \S 102]$.

Normalize the conjugate distance so it equals $\pi$. Then every geodesic is closed and has length $2 \pi$. The mapping of any point into its conjugate point (determined uniquely, independently of the geodesic ray used) is an isometric involution of $M$ onto itself. Any two geodesics intersect in a pair of mutually conjugate points.

Denote the unit tangent bundle of $M$ by $T$, and set $d K=d A d \phi$, where $d A$ is the element of area on $M$ and $d \phi$ is the differential of angle between unit vectors based at the same point. An element of $T$ will be denoted by $e$, or by the pair $(x, \phi)$, where $\phi$ is a fiber coordinate in some local product representation of $T$; set $p(e)=x$. The geodesic flow in $T$ takes $e$ after time $t$ into the element $e_{t}$, the end point of the lift into the bundle of the geodesic segment of length $t$ whose initial element is $e$. It is well known that $d K$, the kinematic density, is invariant under this flow.

Lemma 1.

$$
\int_{T} d K=8 \pi^{2}
$$

Proof. Let a closed geodesic $G_{0}$ divide $M$ into two domains and call the closure of one of them $K_{0}$. Let $G_{\epsilon}$ be the curve swept out by normals to $G_{0}$, pointing a distance $\epsilon$ into $K_{0}$. For sufficiently small

1 This work was supported by a grant of the National Science Foundation. The author wishes to thank J. Adem for first calling this problem to his attention and C. M. Petty for sending him the report [4]. 
$\epsilon>0, G_{\epsilon}$ is a simple closed curve which bounds a geodesically-convex domain $K_{\epsilon}$ interior to $K_{0}$. Set $F_{e}, F_{0}$ equal to the areas of $K_{e}, K_{0}$, respectively, and let $L_{\epsilon}$ be the length of $G_{\epsilon}$. ( $L_{0}=2 \pi$, of course.) Denote by $S_{l, \epsilon}$ the set of elements $e \in T$ such that $p\left(e_{t}\right) \in \bar{K}_{\epsilon}$ for some $t \in[0, l]$. If $l$ is taken less than $\pi$, so the endpoints of the resulting geodesic determine that geodesic uniquely, we may apply the following formula from integral geometry [5, Formula 13.10]

$$
\int_{s_{l, \epsilon}} d K=2 \pi F_{\epsilon}+2 l L_{\mathrm{e}} .
$$

$K_{0}$ is not strictly convex, but both sides of this equation are continuous in $\epsilon$, so we may write $\left(S_{l, 0}=S_{l}\right)$

$$
\int_{S_{l}} d K=2 \pi F_{0}+4 \pi l .
$$

Now we let $l$ approach $\pi$, and notice that every element of $T$ will be in $S_{\boldsymbol{\pi}}$. Hence

$$
\int_{T} d K=2 \pi F_{0}+4 \pi^{2} .
$$

But the left side is just $2 \pi$ times the area of $M$; that is, $4 \pi F_{0}$. Therefore $F_{0}=2 \pi$ and the lemma is proved.

We remark that the validity of the integral geometric formula (1) for this particular situation may also be proved without using approximating curves, essentially by duplicating the proof given by Santaló.

Lemma 2. For any element $e=e_{0}$ in $T$,

$$
\int_{0}^{\pi} R\left(p\left(e_{t}\right)\right) d t \leqq \pi,
$$

and if equality holds $R\left(p\left(e_{t}\right)\right) \equiv 1$ for $0 \leqq t \leqq \pi$.

This result is due to C. M. Petty and James Barry. ([4, Theorem 6.3]; set their $\lambda=\pi$.) It is a local result, in the sense that it involves only the Jacobi equation along the geodesic in question. However, the elegant proof depends on Santaló's in-the-large relations linking the areas within a convex curve and its polar reciprocal [6].

Proof of the theorem. Define

$$
f(e)=\int_{0}^{\pi} R\left(p\left(e_{t}\right)\right) d t .
$$


This is a continuous positive function on $T$. Using the invariance of the measure under the geodesic flow and the Gauss-Bonnet formula, we find that

$$
\begin{aligned}
\int_{T} f(e) d K & =\int_{T}\left[\int_{0}^{\pi} R\left(p\left(e_{t}\right)\right) d t\right] d K=\int_{0}^{\pi}\left[\int_{T} R\left(p\left(e_{t}\right)\right) d K\right] d t \\
& =\int_{0}^{\pi}\left[\int_{T} R(p(e)) d K\right] d t=\pi \int_{0}^{2 \pi}\left[\int_{M} R(x) d A\right] d \phi=8 \pi^{3} .
\end{aligned}
$$

But by the lemmas,

$$
\int_{T} f(e) d K \leqq \pi \int_{T} d K=8 \pi^{3} .
$$

Hence $f(e)=\pi$ for almost all $e$ and the theorem follows from the second assertion of Lemma 2.

In closing, we remark that the device of integrating over $T$ was first used for a problem of this kind by E. Hopf [3].

\section{REFERENCES}

1. W. Blaschke, Vorlesungen über Differentialgeometrie. I, 3te Auflage, Berlin, Springer, 1930.

2. L. W. Green, A sphere characterization related to Blaschke's conjecture, Pacific J. Math. vol. 10 (1960) pp. 837-841.

3. E. Hopf, Closed surfaces without conjugate points, Proc. Nat. Acad. Sci. U.S.A. vol. 34 (1948) pp. 47-51.

4. C. M. Petty, A geometrical approach to the second-order linear differential equation, Lockheed Technical Report LMSD-288250, June, 1960. Amer. J. Math., to appear.

5. L. A. Santal6, Introduction to integral geometry, Paris, Hermann, 1953.

6. Un invariante afin para los cuerpos convexos del espacio de $n$ dimensiones, Portugaliae Math. vol. 8 (1949) pp. 155-161.

Institute of TechNology, University of Minnesota 